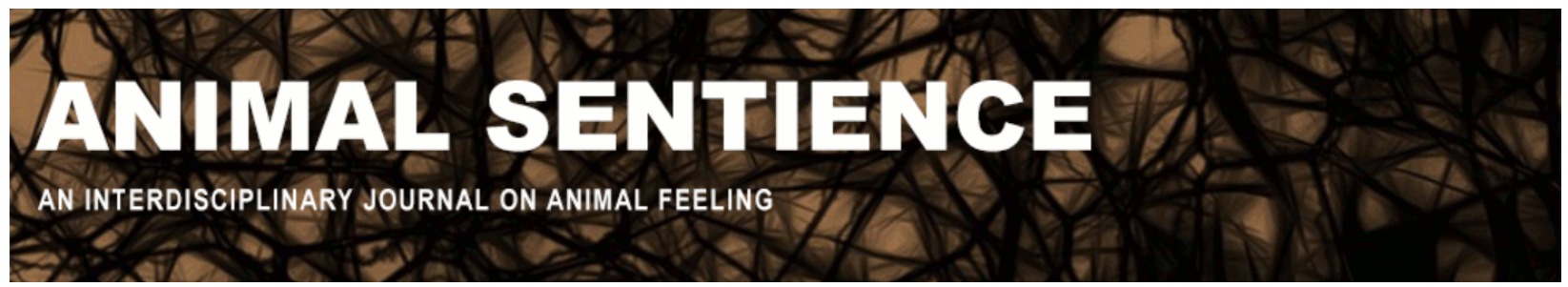

van Hateren, J. H. (2016) Insects have agency but probably not sentience because they lack social bonding. Animal Sentience 9(3)

DOI: $10.51291 / 2377-7478.1130$

Date of submission: 2016-07-28

Date of acceptance: 2016-08-01 (c)

This article has appeared in the journal Animal Sentience, a peer-reviewed journal on animal cognition and feeling. It has been made open access, free for all, by WellBeing International and deposited in the WBI Studies Repository. For more information, please contact

wbisr-info@wellbeingintl.org.

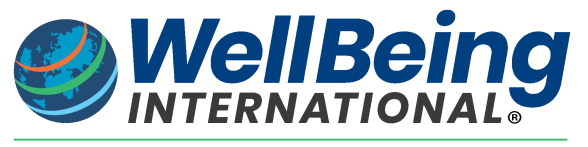

SOLUTIONS FOR PEOPLE, ANIMALS AND ENVIRONMENT 


\title{
Insects have agency but probably not sentience because they lack social bonding
}

Commentary on Klein \& Barron on Insect Experience

\author{
J. H. van Hateren \\ Institute for Mathematics and Computer Science \\ University of Groningen
}

\begin{abstract}
Klein \& Barron (2016) argue that insects have sentience because of functional similarities between the insect brain and vertebrate midbrain. Based on a recent theory of agency and consciousness, I argue that the functional similarities merely point to an advanced form of agency. Insects presumably lack the capacity for social bonding that may be required for subjective experiencing.
\end{abstract}

Hans van Hateren is a faculty member in the Johann Bernoulli Institute for Mathematics and Computer Science at University of Groningen. A biophysicist by training, he has mainly worked on insect vision and computational neuroscience. His current interests focus on the more enigmatic aspects of living organisms, such as agency, goaldirectedness, aboutness, and consciousness. https://sites.google.com/site/jhvanhateren/

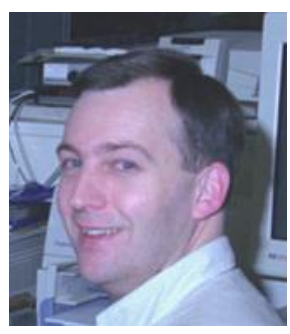

Klein \& Barron (2016; henceforth K \& B) and Barron \& Klein (2016) argue that the central complex in the insect brain has functions similar to those of the midbrain in vertebrates. Both systems integrate information that allows the animal to assess its position in space, to evaluate its own state and that of its environment, and thereby to select relevant actions. Earlier, Merker (2007) argued that the midbrain is the source of subjective experience in vertebrates because of the integrated, egocentric perspective on the world that it provides. K \& B conjecture that the insect brain may produce subjective experience similarly, as it appears to enable a similar integrated perspective.

There is much to like in $\mathrm{K} \& \mathrm{~B}^{\prime} \mathrm{s}$ target article. They assume that there is a primordial, lightweight form of consciousness, a mere sense of subjective experiencing (sentience). This is a quite reasonable assumption, in my view. They also argue that the smallness of insect brains is not a good argument against the existence of simple forms of consciousness. I agree with that too: insect brains may be small, but they are quite sophisticated, and it is not known how much complexity is actually needed for consciousness. Finally, K \& B argue convincingly that there are indeed close functional parallels between the insect and vertebrate nervous systems.

Nevertheless, I do not follow their conclusion that insects are indeed capable of subjective experience, for two main reasons. First, for a system-theoretic reason, and second, because I see a better explanation of the facts they review. Both reasons also apply, to some extent, to Merker (2007).

The system-theoretic reason boils down to the fact that what $\mathrm{K} \& \mathrm{~B}$ describe is a straightforward cybernetic system. One could readily build a robotic system that tracks its own position in space, tracks its own state and that of the environment with respect to its goals, and 
that uses this for selecting actions. Although such a system might become quite complex in a complex, natural environment, it would not require anything special. The entirety of the system would give the robot a unique, egocentric view on the world, which is exactly what $K \& B$ (p. 3) conjecture. But it is not clear to me why such a system would produce subjective experience. Where would that come from? What causal role would it have? The system would function perfectly well without it, as a regular physico-chemical system.

The above paragraph should not, however, be taken as an argument that insects are mere automata (functioning mechanically and involuntarily). I think they are not, because they clearly have agency. This brings me to the alternative explanation I mentioned. In addition to subjective experience, there is a more basic "hard" problem in living organisms, which equally evades straightforward functional and computational modeling. This problem is the fact that individual organisms have intrinsic goals, act on their own behalf, and can choose their own course of action, at least to some extent. In humans, such phenomena are traditionally described by high-level terms such as purpose, intentionality, agency, and free will. But simpler variants of these phenomena presumably exist in all living organisms, and one can indeed observe them easily in many animal species. Automata do not have such properties, so where do they come from?

I have recently developed a theory that explains how agency and goal-directedness can be generated (van Hateren 2015a). The theory is supported by computational as well as mathematical analysis (van Hateren 2015b). I will try to explain it briefly here, in general terms. The theory assumes that the actual evolutionary fitness of an organism (that is, its capacity for surviving and procreating) is tracked, in real time, by an internal approximation of this fitness. The approximation is produced by physiological or neuronal processes within the organism. It is part of a highly specific control loop that utilizes randomness in a targeted way to change behavior. The total system can then be shown to be evolvable. Remarkably, it produces, at one stroke, primordial forms of agency, goal-directedness, and aboutness (the property that, e.g., specific neuronal processes can be about specific external processes). In effect, it generates a novel causal factor of a type that is absent from the non-living world. This factor is similar to a correlation, but a rather special one. Normal correlations are only mathematical tools for understanding how things are related. They are not causal factors by themselves, but just describe the statistics produced by the actual, underlying causal factors. In contrast, the correlation between approximated and actual fitness has causal effectiveness of its own (van Hateren 2015b). Its effectiveness cannot be derived from or reduced to underlying factors. Importantly, it produces goals that are intrinsic to the organism, not dependent on external agency. In contrast, a control system, such as a thermostat, has no intrinsic goal. It is just a device serving an external goal, such as someone's goal of keeping the house warm.

The above theory of agency fits nicely with the functional roles ascribed to the insect brain by K \& B and to the midbrain by Merker (2007). By producing an evaluative, egocentric perspective on the world, these brain structures provide the animal with an essential component for evaluating and using its fitness approximation. Depending on this evaluation, targets and actions can be varied and selected. This type of agency could not be implemented by an automaton, because of the way agency depends on randomness and fitness. Automata do not have a fitness in the biological sense (which requires the potential of exponential growth in numbers as a result of descent with modification). 
So where does subjective experience enter this picture? Agency and aboutness as such would not be enough to produce it, because unicellular organisms and plants presumably also have basic variants of these. The most plausible evolutionary point of origin I see is the point (or points) where agency and aboutness became shared between individuals. Sharing agency and aboutness requires, roughly speaking, bonding between organisms (of the kind that is common in mammals and birds, at least). Bonding and nonverbal dialogue then continually modify the internal fitness approximation of each bonded partner, such that it runs partly through the approximation of the other. Thus, goals, agency, and aboutness become dynamically shared between individuals. An external partner is not necessary when the nonverbal dialogue becomes internalized, with a virtual or abstract partner. I conjecture that the complex causal novelty formed by shared agency is manifest as subjective experience (van Hateren 2015a). The main reason for this conjecture is that sharing agency requires transforming an unconventional non-material cause (i.e., the agency/goals/aboutness complex) into the conventional material causes that are necessary for dialogue (e.g., touch, postures, and sounds). A very strange transformation indeed. The consequence of the dialogue is a change of the organism's internal fitness approximation. The latter represents all that the organism implicitly values, hence its change may explain why this is felt by the organism.

The proposed close connection between agency and consciousness is consistent with the claim that at least some variants of the vertebrate midbrain are involved in producing consciousness (Merker 2007). This might depend on a species-dependent capacity for sharing agency and aboutness. However, I am not aware of studies reporting dynamical social bonding in insects, including the social insects. If this absence of social bonding in insects is empirically correct, and if the above theory of agency and consciousness holds, then we can conclude as follows: $K \& B$ have made the point that insects have the capacity for advanced agency, but not that they have the capacity for subjective experience.

\section{References}

Barron, A. B., \& Klein, C. (2016). What insects can tell us about the origins of consciousness. Proceedings of the National Academy of Science of the United States of America, 113, 49004908.

Klein, C., \& Barron, A. B. (2016). Insects have the capacity for subjective experience. Animal Sentience 2016.100.

Merker, B. (2007). Consciousness without a cerebral cortex: A challenge for neuroscience and medicine. Behavioral and Brain Sciences, 30, 63-81.

van Hateren, J. H. (2015a). The origin of agency, consciousness, and free will. Phenomenology and the Cognitive Sciences, 14, 979-1000.

van Hateren, J. H. (2015b). Causal non-locality can arise from constrained replication. EPL Europhysics Letters, 112, 20004. 